\title{
Genome-wide detection and sequence conservation analysis of long non-coding RNA during hair follicle cycle of yak
}

Xiaolan Zhang, Qi Bao, Congjun Jia, Chen Li, Yongfang Chang, Xiaoyun Wu, Chunnian Liang, Pengjia Bao* and Ping Yan $^{*}$ (D)

\begin{abstract}
Background: Long non-coding RNA (IncRNA) as an important regulator has been demonstrated playing an indispensable role in the biological process of hair follicles (HFs) growth. However, their function and expression profile in the HFs cycle of yak are yet unknown. Only a few functional IncRNAs have been identified, partly due to the low sequence conservation and lack of identified conserved properties in IncRNAs. Here, IncRNA-seq was employed to detect the expression profile of IncRNAs during the HFs cycle of yak, and the sequence conservation of two datasets between yak and cashmere goat during the HFs cycle was analyzed.
\end{abstract}

Results: A total of 2884 IncRNAs were identified in 5 phases (Jan., Mar., Jun., Aug., and Oct.) during the HFs cycle of yak. Then, differential expression analysis between 3 phases (Jan., Mar., and Oct.) was performed, revealing that 198 differentially expressed IncRNAs (DELs) were obtained in the Oct.-vs-Jan. group, 280 DELs were obtained in the Jan.vs-Mar. group, and 340 DELs were obtained in the Mar.-vs-Oct. group. Subsequently, the nearest genes of IncRNAs were searched as the potential target genes and used to explore the function of DELs by GO and KEGG enrichment analysis. Several critical pathways involved in HFs development such as Wnt signaling pathway, VEGF signaling pathway, and signaling pathways regulating pluripotency of stem cells, were enriched. To further screen key IncRNAs influencing the HFs cycle, 24 DELs with differ degree of sequence conservation were obtained via a comparative analysis of partial DELs with previously published IncRNA-seq data of cashmere goat in the HFs cycle using NCBI BLAST-2.9.0+, and 3 DELs of them were randomly selected for further detailed analysis of the sequence conservation properties.

Conclusions: This study revealed the expression pattern and potential function of IncRNAs during HFs cycle of yak, which would expand the knowledge about the role of IncRNAs in the HFs cycle. The findings related to sequence conservation properties of IncRNAs in the HFs cycle between the two species may provide valuable insights into the study of IncRNA functionality and mechanism.

Keywords: Hair follicle cycling, IncRNA, NCBI blast-2.9.0 +, Yak

\footnotetext{
*Correspondence: baopengjia@caas.cn; pingyanlz@163.com

Key Laboratory of Yak Breeding Engineering Gansu Province, Lanzhou Institute of Husbandry and Pharmaceutical Sciences, Chinese Academy of Agricultural Sciences, Lanzhou 730050, China
}

(c) The Author(s). 2020 Open Access This article is licensed under a Creative Commons Attribution 4.0 International License, which permits use, sharing, adaptation, distribution and reproduction in any medium or format, as long as you give appropriate credit to the original author(s) and the source, provide a link to the Creative Commons licence, and indicate if changes were made. The images or other third party material in this article are included in the article's Creative Commons licence, unless indicated otherwise in a credit line to the material. If material is not included in the article's Creative Commons licence and your intended use is not permitted by statutory regulation or exceeds the permitted use, you will need to obtain permission directly from the copyright holder. To view a copy of this licence, visit http://creativecommons.org/licenses/by/4.0/ The Creative Commons Public Domain Dedication waiver (http://creativecommons.org/publicdomain/zero/1.0/) applies to the data made available in this article, unless otherwise stated in a credit line to the data. 


\section{Background}

Yak (Bos grunniens) is the only domesticated breed in the Bos genus that can produce wool. It has been regarded as "all-purpose" livestock to provide essential living and economy resource for inhabitants around the Qinghai-Tibetan Plateau. The hair growth cycle of yak is similar to that of cashmere goat, also presenting a seasonal pattern under the control of the animals' endocrine systems and photoperiod, including anagen (growth), catagen (regression), and telogen (rest) [1, 2]. The morphological changes in hair growth take place following different phases of the HF cycle [3]. In the anagen phase, an entire hair shaft from follicles is produced, this phase determines the length of the hair shaft. The catagen phase is the dynamic transition from the anagen phase to the telogen, in which the HFs regress in a lower cell cycling process caused by the increased apoptosis of epithelial cells in the bulb, outer root sheath, and outermost epithelial layer. During the telogen phase, follicles regenerate with the activation of multipotent stem cells, receiving signals to initiate a new cycle of hair growth [4]. The transition between the phases is the result of multiple molecular signals and their intricate interactions in the skin during HF development. Several signaling pathways, such as Wnt, Notch, Hedgehog, and bone morphogenetic protein (BMP), are crucial for the regulation of HFs cycle [5]. Moreover, some hormones and molecules, such as melatonin, estrogen, fibroblast growth factor 5 (FGF5), vascular endothelial growth factor (VEGF), and so on, have been identified influencing the development of the HFs cycle [6-9].

An increasing number of studies have shown that long non-coding RNAs (lncRNAs) play vital roles in regulating various biological processes, such as development, cell proliferation, and differentiation [10, 11]. LncRNAs regulate gene expression at various genomic levels, including chromatin modification, transcription, and posttranscriptional regulation, and multiple binding modules are involved that affect the gene expression in the immediate genomic vicinity (in cis) or at other genomic locations (in trans) [12-14]. LncRNAs are crucial for maintaining pluripotency and lineage commitment [15]. In dermal papilla, lncRNAs regulate the gene expression associated with HFs development and postnatal hair cycling [16]. In addition, lncRNAs have been found to participate in the regulation of the HFs cycle in cashmere goats, sheep and Angora rabbit, besides regulating skin pigmentation in goats and cattle [17-20]. So far, the studies on HF growth in yak focused mainly on the expression pattern of several genes such as BMP2, TGF- $\beta$ and HSP7O [21-23]. No systematic molecular study reported on the HFs cycle in yak. The hair growth pattern of yak is similar to that of goats. Also, the harsh living environment with cold and oxygen-thin air gives yak some unique traits, such as the high altitude adaptation [24]. The skin is an important protective barrier against a cold and harsh environment. Studies on the influence of lncRNAs on HFs may broaden the knowledge on the alpine adaptation of yak.

The functions of lncRNAs were predicted by analyzing the role of their potential targets in cis or trans co-localization or correlation. Only a small fraction of lncRNAs have been experimentally tested for their function [12, 25], including the well-studied lncRNAs Xist, HOTAIR, and MALAT1 that were synchronously reported to have higher sequence conversation or a conservative domain [26-28]. In fact, contrary to coding genes, lncRNAs were mostly reported with a lower level of sequence conservation and lacked identified conservation properties across species [12], hindering the studies on lncRNAs function. The findings about lncRNAs in position and evolution conservation are helpful to further understand their roles in various biological processes. LncRNAs were found to be ancient components in the evolution of vertebrate genome and showed an unprecedented evolutionary plasticity, and the lncRNAs conserved in position were primarily connected to many developmental transcription factors $[29,30]$. These findings indicated the importance of lncRNAs in genome evolution and development from the view of functional commonality. However, the functional exploration of individual lncRNAs needed to be performed with elaborate analysis. Recent studies demonstrated the secondary structure conservation of homologous lncRNAs among species [13], highlighting the significance of structure in functionality of lncRNAs, along with species-specific and spatiotemporal expression patterns [31]. The sequence conservation of lncRNAs for the same trait at the interspecies level is worth exploring. The growth pattern of HFs in yak and cashmere goat provided a good animal model for the sequence conservation study of lncRNAs in the same trait.

The objective of this study was to investigate the expression pattern of lncRNAs during HFs cycle of yak and detect the potential function of lncRNAs involved. The sequence conservation of partial lncRNAs between yak and cashmere goat during HFs cycle was analyzed to obtain more critical lncRNAs associated with the HFs cycle of yak. The present study provided a systematic knowledge about lncRNAs regulating the HFs cycle of yak and screened lncRNAs that were sequence-conserved between yak and goat, thus providing insights into the role of lncRNAs in hair growth biology and laying a foundation for further functional studies. 


\section{Methods}

\section{Animals and sample collection}

Tianzhu white yaks with fine fiber production trait were used in this study. All the yaks were obtained from the Tianzhu white yak propagation bases of Gansu province, China. Female yaks about 2 years old in similar shape (coefficient of relationship <0.125) were selected for sample collection in different phases of the HFs cycle that included the following five time points: Jan., Mar., Jun., Aug., and Oct.. Skin samples of 3 yaks at each point were collected from the scapulae region using a medical skin sampler after local injection of $2 \%$ lidocaine and rapidly frozen in liquid nitrogen for further processing. Veterinary streptomycin sulfate and penicillin potassium powders were applied to the wound and put a sterile dressing over the wound. The animal wounds can heal completely within a month. The samples were collected at the adjacent site in each distinct phase.

All the experimental procedures involved in this study were approved by the Animal Management and Ethics Committee of the Lanzhou Institute of Animal Science and Veterinary Medicine, Chinese Academy of Agricultural Sciences (Permit No. SYXK-2016-0039).

\section{RNA isolation, library preparation, and sequencing}

Total RNA of skin tissues was extracted using TRIzol reagent (Invitrogen, CA, USA) following the manufacturer's protocols, after grinding them in liquid nitrogen. RNA integrity was analyzed using an RNA Nano 6000 Assay Kit of the Bioanalyzer 2100 system (Agilent Technologies, CA, USA). RNA concentration and purity were measured with the Nanodrop 2000 photometer spectrophotometer (Implen, CA, USA). Approximately $3 \mu \mathrm{g}$ RNA was used for RNA library preparation. First, ribosomal RNA was removed using TruSeq Stranded Total RNA with a Ribo-Zero Gold Kit (Illumina, CA, USA). Subsequently, interruption reagent was added to break the rRNA-depleted RNA into short fragments, and the latter RNA was used as a template. Random hexamer primers were used to synthesize the first-strand cDNA, and then the second-strand cDNA was synthesized using DNA polymerase I and RNase $H$. In the reaction system of second-strand cDNA, dUTP was used instead of dTTP. Then, different adapters were connected, and the UNG enzyme method was used to digest the strand containing dUTP, only retaining the first cDNA strands with different linkers. After purification of the cDNA strands, the remaining overhangs were converted into blunt ends via polymerase/exonuclease activities, and adenylation of the 3 ' ends were performed. The sequencing adapter was ligated and the cDNA fragments of 150-200 bp in length were selected to perform PCR amplification. Finally, the PCR products were purified (AMPure XP system), and RNA library quality was assessed with an
Agilent 2100 Bioanalyzer system (Kapa BioSystems, MA, USA). The clustering of the index-coded samples was performed using TruSeq PE Cluster reagent (Illumina, CA, USA) on a cBot Cluster generation system following the manufacturer's protocols. Then, the cDNA libraries were subjected to standard paired-end sequencing with an Illumina Hiseq 2500 platform and $150 \mathrm{bp}$ paired-end reads were generated.

\section{Quality control and transcriptome assembly}

Raw reads were trimmed by removing adapter sequences, reads containing over $10 \%$ of poly $-\mathrm{N}$, and lowquality bases ( $>50 \%$ of bases whose Phred scores were < 5\%) using in-house Perl scripts. The valid bases, Phred score (Q30) and GC content were used to filter high quality clean data. The alignment of paired-end clean reads to the reference genome (versionGCA 005887515.2 BosGru3.0) was performed using the hisat2 [32]. The mapped reads of each library were assembled using Cufflinks (v2.1.1) in a reference-based approach [33]. For the prediction of lncRNAs, reassembling of transcripts was performed using a streaming neural network algorithm with StringTie software [34], which could accurately determine the direction of transcript chains for the data of chain-specific library.

\section{Identification of IncRNAs}

Based on the characteristics of lncRNAs, a strict fourstep procedure was used to identify lncRNAs from the assembled transcripts: (1) spliced transcripts were compared with reference transcripts using Cuffcompare v2.1.1 [33] to remove known coding transcripts or loci, and the location type of the remaining transcripts was determined; (2) transcripts with length $<200 \mathrm{bp}$ or exon number $<2$ were removed; (3) the coding potential of transcripts was analyzed to remove transcripts with coding potential. The analytic software was CNCI (v2) [35], CPC (0.9-r2) [36], Pfam-scan (v1.3) [37], and PLEK [38]. (4) LncRNA transcripts obtained in the third step were used to align the annotated lncRNAs in yak with Blastn software, the transcripts overlapped with known lncRNAs of yak were removed, and novel lncRNAs were screened. Then, novel lncRNAs and known lncRNA transcripts were used for further quantitative analysis. FEELnc [39] was used to classify and count the different types of identified novel lncRNAs based on the positional relationship with their closest protein coding transcripts; the classification involved four sides including direction (sense or antisense), type (genic or intergenic), location (e.g. upstream for lincRNA or exonic for genic lncRNAs), and subtype (e.g. divergent for lincRNAs or containing for genic lncRNAs). 


\section{Differential expression analysis of IncRNA}

Fragments per kilobase for a million reads (FPKM) of lncRNA transcripts was calculated with Cuffdiff (v2.1.1) [40]. DESeq [41] was used to normalize the lncRNA counts of each skin sample (basemen value to estimate the expression). $P$ value $<0.05$ and foldchange $>2$ was set as the threshold for significantly differential expression between any two distinct phases of HFs growth. Based on the count data of lncRNA expression, sample similarities of the three groups (Jan., Mar., and Oct.) was analyzed via principal component analysis (PCA). Besides, heatmap showing the Euclidean distance between the samples as calculated from the variance stabilizing transformation of the count data, also was used to check the consistency of the biological replicates.

\section{Function enrichment analysis of the nearby genes of differential IncRNAs}

The nearest protein-coding gene paired with each lncRNA was searched as the potential regulatory target gene of that lncRNA. If no gene was detected within $100 \mathrm{~kb}$ upstream or downstream of a lncRNA, that lncRNA was excluded in subsequent analysis. Function enrichment analysis of the target genes between any two groups DELs were performed, using the clusterProfiler $\mathrm{R}$ package depends on gene ontology (GO) database and the Kyoto Encyclopedia of Genes and Genomes (KEGG) database, to calculate enrichment test for GO terms and KEGG pathways based on hypergeometric distribution [42]. The protein-protein interaction network of all the nearby genes paired with DELs was analyzed with the STRI NG database (https://string-db.org/), and further visualized with Cytoscape V3.5.1 (http://www.cytoscape. $\operatorname{org} /)$.

\section{Verification of sequencing data using real-time quantitative PCR}

Total RNA from the skin tissue subjected to RNA sequencing was also used to verify the sequencing results by real-time quantitative PCR (RT-qPCR). The first-strand cDNA was obtained using a PrimeScript RT reagent kit with gDNA Eraser (TaKaRa, Dalian, China). The RT-qPCR was performed using SYBR Premix Ex Taq II (TaKaRa) on the Bio-Rad CFX96 TouchReal-Time PCR Detection System (Bio-Rad, CA, USA). The reaction conditions were set as follows: $95^{\circ} \mathrm{C}$ for $3 \mathrm{~min}$, followed by 40 cycles of $95^{\circ} \mathrm{C}$ for $12 \mathrm{~s}$ and $60^{\circ} \mathrm{C}$ for $30 \mathrm{~s}$. The relative expression of lncRNAs and mRNAs was analyzed with the $2^{-\Delta \Delta C t}$ method and normalized using GAPDH. The primers used for RT-qPCR were designed with oligo 6 and are listed in Additional file 1.
Sequence conservation analysis of IncRNAs between yak and cashmere goat during HFs cycle

To further screen the key lncRNAs in the HFs cycle of yak, DELs paired with differently expressed genes (DEGs) were screened by comparing analysis the lncRNA data with our previous mRNA data [43]. Then, these DELs were used for sequence conservation analysis with the lncRNA data of cashmere goat during the HFs cycle using NCBI BLAST-2.9.0+ [44] with parameters of "Gap Penalties: Existence 0, Extension 2.5, Expectation value 1e-10". The lncRNA dataset of the cashmere goat was used as "blastdb database", and the DELs of the yak were used as "query database". The lncRNA dataset of the cashmere goat was downloaded from a previous study [19]. The sequence conservation properties were analyzed using dottup website, WebLogo3 (http:// weblogo.threeplusone.com/), Clone Manager bioinformatics software, and so forth.

\section{Results \\ Identification and differential expression analysis of IncRNAs in the yak skin}

A total of $748.61 \mathrm{M}$ raw reads were produced from 15 skin samples in 5 phase groups of yaks using the Illumina Hiseq 2500 platform, in which $733.79 \mathrm{M}$ reads were clean (Additional file 2), accounting for $98 \%$ of raw reads. After screening with rigorous criteria and analyzing the coding potential with the software CNCI, CPC, Pfam and PLEK, 2884 lncRNAs from all samples were identified (Fig. 1a). Of these transcripts, $70.1 \%$ (2023) were novel lncRNAs. The average length of novel lncRNAs was $1152 \mathrm{bp}$ (Fig. 1b). Furthermore, the data showed that approximately $40 \%$ of the novel lncRNAs were antisense lncRNAs and $60 \%$ were sense lncRNAs (Fig. 1c), besides approximately $90 \%$ of the novel lncRNAs with two to three exons (Fig. 1d). These data of lncRNAs were similar to the features of previously described lncRNAs [45].

The expression levels of lncRNAs were analyzed using Cuffdiff v2.1.1. The DELs of every two distinct groups were screened with the threshold set as fold change $>2$ and $p$ value $<0.05$ (Additional file 3 ). Numbers of all the DELs between every comparison groups, and the common and specific DELs were visualized and were shown in Fig. $1 \mathrm{e}$ and $\mathrm{f}$. Based on the result, Oct. was selected in the anagen phase with Jan. (catagen) and Mar. (telogen) for further functional analysis, because both Mar.-vs-Oct. and Jan.-vs-Oct. comparison groups had a higher number of DELs, while the number of DELs was relatively minor in the Mar.-vs-Jun. and Aug.-vs-Oct. comparison groups. Jun. and Aug. were preliminarily excluded to simplify subsequent analysis. In the differential expression analysis of the 3 groups (Jan., Mar. and Oct.), 280 DELs were obtained in the Jan.-vs-Mar., 340 and 198 DELs were obtained in the Mar.-vs-Oct. and Jan.-vs-Oct. comparison 
a

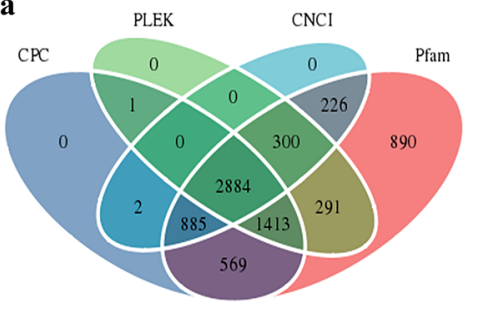

c

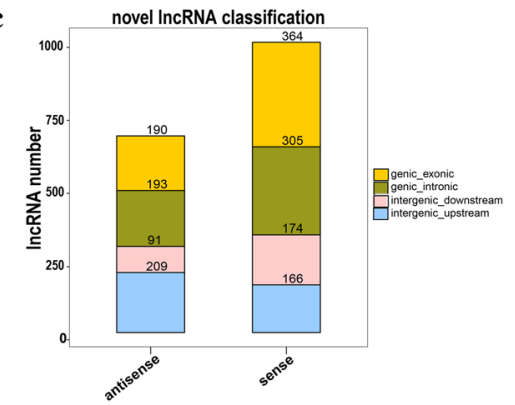

e Statistic of Differently Expressed IncRNA

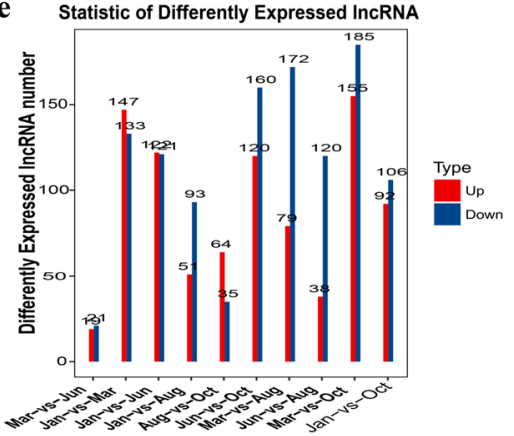

f

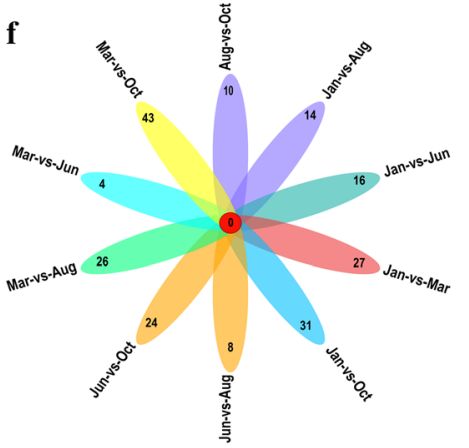

b

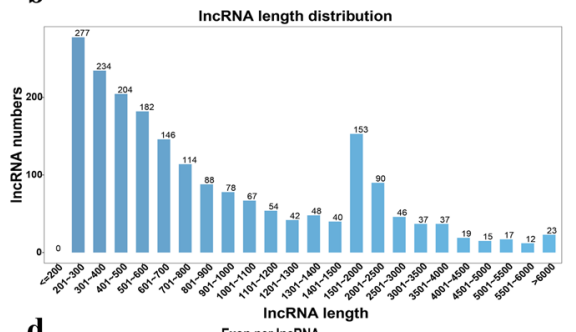

d Exon per IncRAA
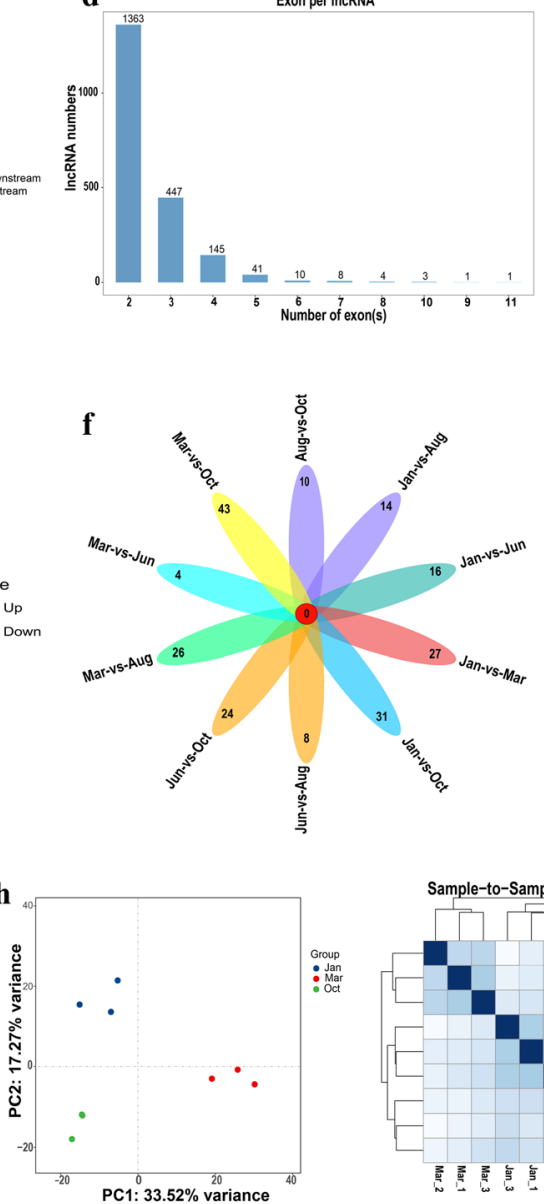

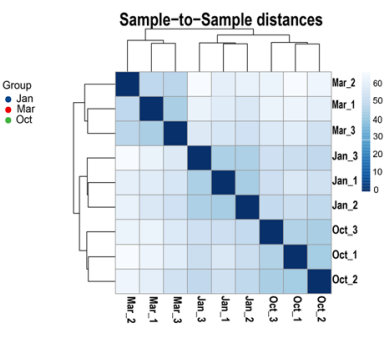

Fig. 1 Long noncoding RNAs (IncRNAs) identification and comparative analysis of their differential expression in the hair follicles (HFs) cycle of yak. a Screening of the candidate IncRNAs in skin transcriptome. Four tools (CPC, PLEK, CNCl, and PFAM) were employed to analyze the coding potential of IncRNAs. Those IncRNAs shared by the four analytical tools were designated as candidate IncRNAs and used for subsequent analysis. b The length distribution, c classification, and $\mathbf{d}$ exon number distribution of novel IncRNAs. e Statistical analysis of differently expressed IncRNAs in every two comparison groups. $\mathbf{f}$ The common and specific differently expressed IncRNAs between different comparison groups (right). $\mathbf{g}$ Venn diagrams of DELs in three comparison groups, including Jan.-vs-Mar., Jan.-vs-Oct. and Mar.-vs-Oct., the IncRNAs shared with the three groups indicated differently expressed in every comparison group. $\mathbf{h}$ PCA and the analysis of sample-to-sample distance were used to check the sample similarities of the three groups (Jan., Mar. and Oct.)

groups, respectively. Also, 22 of these DELs were shared in the 3 comparison groups (Fig. 1g). PCA was used to analyze the sample similarities. A more concentrated distribution of samples in the same group was clearly observed. In addition, Fig. $1 \mathrm{~h}$ showed that the sample-tosample distances are closer in the same group. The data indicated that the samples had high reliability.

\section{GO and KEGG enrichment analysis}

The nearest protein-coding genes paired with DELs were used to perform GO and KEGG enrichment analysis so as to investigate the potential function of DELs during the three distinct HFs phases. Figure 2a shows the top 30 GO terms (top 10 terms in each GO category). The result showed that the GO terms were enriched mainly 


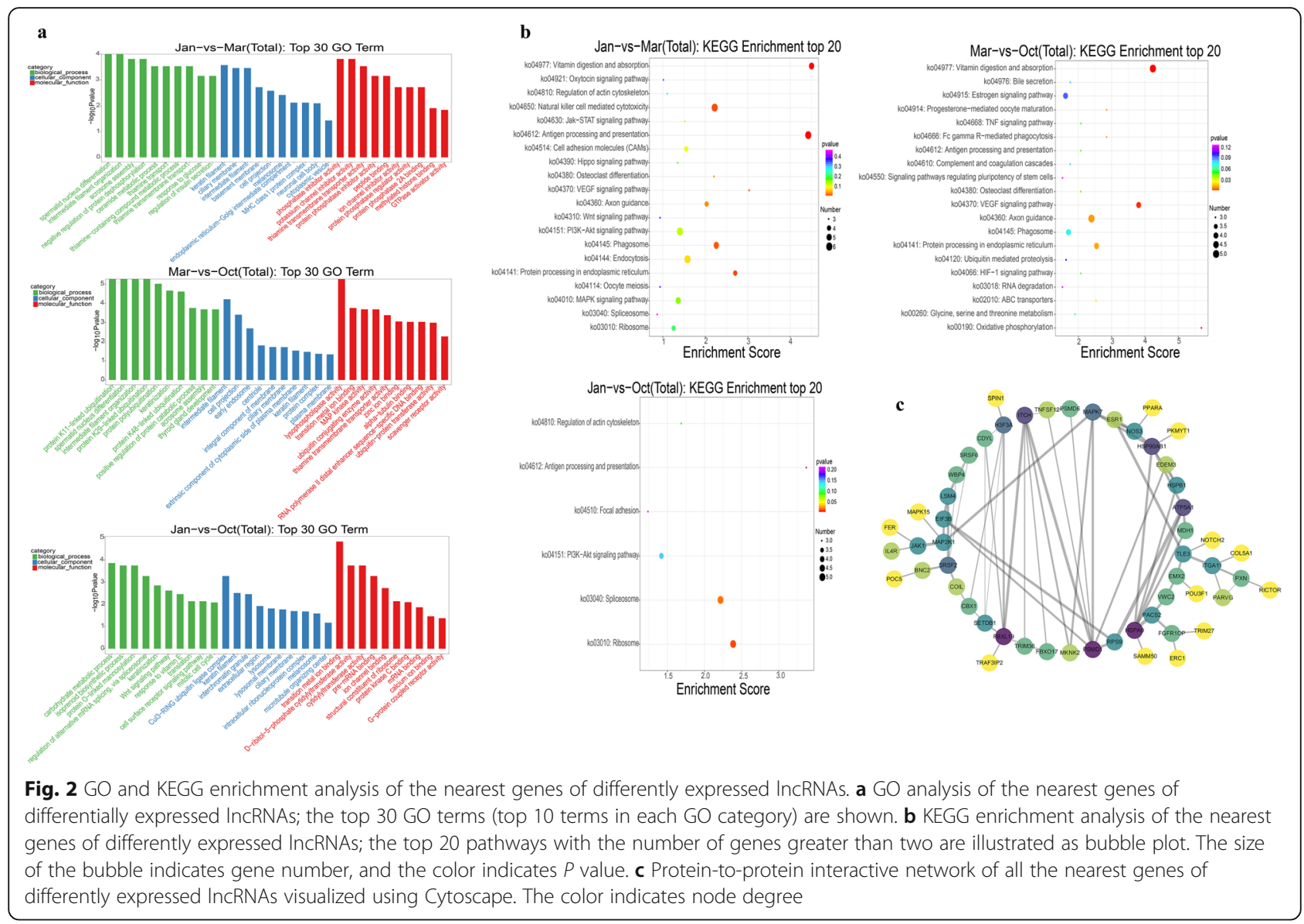

in Keratinization, keratin filament, carbohydrate metabolic process, and thiamine transport, which are involved in hair formation or nutrient metabolism. In the Mar.-vs-Oct. comparison group, protein ubiquitination was highly enriched. The ubiquitin-mediated proteolysis pathway was reported to be important for distinguishing the secondary hair follicles (SFs) from primary hair follicles (PFs) in cashmere goats [46, 47], suggesting that the regulation of protein ubiquitination was related to the transition between PFs and SFs from Mar. to Oct. in the present study.

KEGG enrichment analysis was performed. The top 20 KEGG pathways with the number of DELs greater than 2 are shown in Fig. 2b. The VEGF signaling pathway, Wnt signaling pathway, and signaling pathways regulating the pluripotency of stem cells, which were reported to play indispensable roles during the HFs cycle, were enriched [48-50]. In addition, hormone and nutrition metabolism signaling pathway (e.g. estrogen signaling pathway, vitamin digestion and absorption, and glycine, serine, and threonine metabolism), immune response signaling pathway (e.g. antigen processing and presentation and natural killer cell-mediated cytotoxicity) were enriched, which might be essential for the HFs growth $[6,51]$. The pathways among distinct phases coincidentally corresponding with the biological process of hair cycling seemed to orchestrate a dynamic genesis of the HFs cycle at the molecular level. For example, in Jan.-vs-Mar. comparison group, the only upregulated pathway was vitamin digestion and absorption due to the relative resting state in the telogen (Mar.) and reserve energy for the next HFs cycle (Figure S1). Moreover, the HIF-1 signaling pathway involved in the perception of hypoxia was enriched, which might be related to the high-altitude environment with frigid and hypoxia of yak living. The protein-protein interaction networks of coding genes that paired with all the DELs of the three distinct phases were analyzed using the STRING database (https://string-db.org). The result showed that ITCH, PSMD1, FBXL19, and HSPA9 primarily involved in protein ubiquitination (queried from the UniProt database) were key nodes of the DELs paired genes (Fig. 2c). The ubiquitin-mediated proteolysis pathway was important for distinguishing SFs from PFs in cashmere goats $[46,47]$, and it was recently reported that the ubiquitylation process associated with 
Hedgehog signaling a crucial signaling that affecting on HFs regeneration [52-54].

\section{Comparative analysis of DELs in yak with cashmere goat IncRNA data}

To further screen the DELs that are more likely to affect the HFs cycle in yak, 93 differently expressed protein coding genes (DEGs) paired with 110 DELs were obtained by combining the present data with our previous mRNA data on the HFs cycle in yak [43]. The detailed information on these DELs and DEGs were presented in Additional file 4. The expression tendency and abundance of most DELs were consistent with the paired coding genes, although a few pairs such as TCONS 00055063 with its paired gene NOS3 had an opposite expression tendency. Six lncRNAs and four lncRNA-paired protein-coding genes, which were differentially expressed in at least any two distinct phases, were selected to verify the transcriptome sequencing data by RT-qPCR. LncRNAs were selected based on the expression levels and potential function of the paired proteincoding gene in hair development. Figure 3a shows the RT-qPCR result and the comparison of the sequencing data and RT-qPCR data.

LncRNAs have species and tissue specificity with lower sequence conservation [11]. Yak and cashmere goat belong to the Bovidae family of ruminants, and they had a similar seasonal HFs cycle. To explore the sequence conservation level between yak and cashmere goat during the HFs cycle and obtain more reliable DELs regulating HFs growth, NCBI BLAST$2.9 .0+$ was used to detect the sequence-conserved
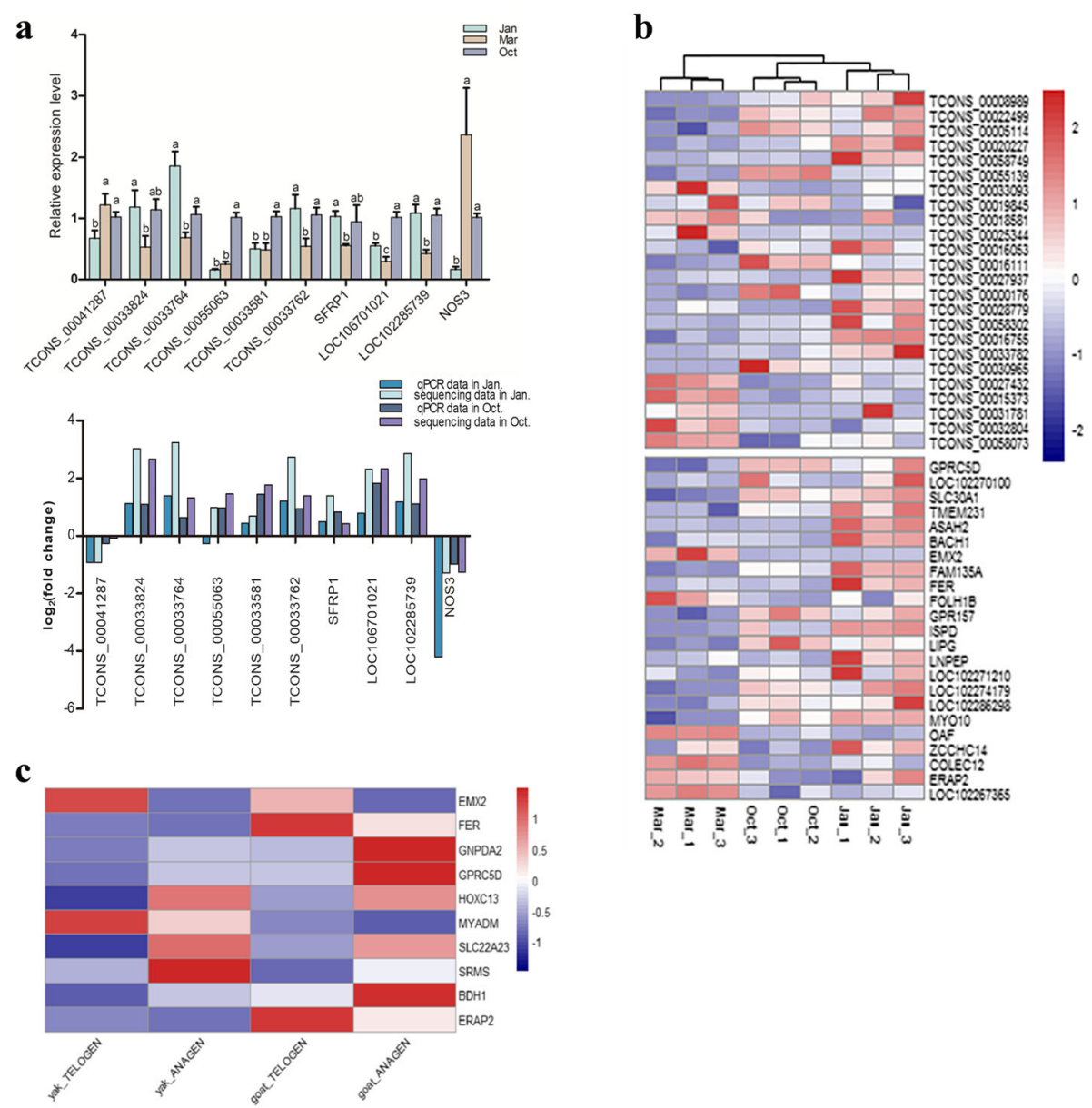

Fig. 3 Verification of sequencing data by RT-qPCR and screening of sequence conserved DELs between yak and cashmere goat. a The expression level of differently expressed IncRNAs and several paired DEGs were detected by RT-qPCR (above), the relative expression levels of IncRNAs and mRNAs were analyzed by the $2^{-\Delta \Delta C t}$ method and normalized using GAPDH. Data were presented as means $\pm \operatorname{SEM}(n=3)$. The same letter means no significant difference, different letters mean significant difference; Comparation of the expression pattern of the sequencing data and RT-qPCR data (below). Log2(fold change) $>0$ indicates the transcript up regulated in Jan. (catagen) or Oct. (anagen) compared to Mar.(telogen). Log 2 (fold change) $<0$ is the opposite. $\mathbf{b}$ Cluster analysis of the aligned 24 DELs and their paired 23 differently expressed mRNAs in yak. $\mathbf{c}$ Heat map showing the expression profiles of DEGs in both yak and cashmere goat during the HFs cycle 
lncRNAs of these 110 DELs with the lncRNA data of the cashmere goat in the HFs cycle (532 lncRNA sequences) [19]. Sequences information of all the lncRNAs of yak in this study was showed in Additional file 5. The BLAST result found that 24 DELs among these 110 DELs of yak had sequence identities with cashmere goat lncRNA data to different degrees. Then, the expression profiles of aligned DELs in the yak with their respective alignments in the cashmere goat dataset were comparatively analyzed. Excluding the repetitive lncRNA sequences, a total of 287 alignments including 125 lncRNA sequences in the cashmere goat dataset were aligned by 24 DELs of the yak dataset (Additional files 6 and 7). The aligned lncRNA sequences in both datasets all accounted for approximately 20\% (125/532, 24/110) of their total databases used in BLAST2.9.0+ (Additional file 6). Among the 125 lncRNA sequences, 41 were found to be differentially expressed in the cashmere goat during the HFs cycle [19]. Comparative analysis found that the expression profiles of most of the differently expressed alignments (approximately 80\%) in the cashmere goat in anagen and telogen were similar to those of the aligned DELs in the yak in anagen (Oct.) and the transition from catagen (Jan.) to telogen (Mar.) (Additional file 8). This finding suggested that the number of lncRNAs with a conserved sequence is limited, accounting for about $20 \%$. The expression pattern of the sequence conserved DELs between the yak and cashmere goat data had a little difference, which probably related to the difference from species and sampling time, considering only two phases of the HFs cycle in the cashmere goat. Or not all kinds of conserved elements in the lncRNAs play the similar roles between species. Further experiments are needed to detect whether the DELs with conserved elements between the yak and cashmere goat playing similar functions during the HFs cycle.

Figure $3 \mathrm{~b}$ shows the 24 DELs and their paired 23 DEGs of the yak with a heat map. In addition, comparative analysis of the 93 DEGs in the yak with the DEGs between the anagen and telogen of cashmere goat data [19] found that 10 DEGs were differentially expressed both in the yak and cashmere goat during the HFs cycle and the expression tendency in the cyclic process was consistent (Fig. 3c). The paired lncRNAs of genes GPRC5D, FER, EMX2 and ERAP2 simultaneously had a conserved sequence between the yak and cashmere goat. GPRC5D and EMX2 were associated with HFs morphogenesis $[55,56]$. These results verified the sequencing data and further revealed some potentially important DELs and their paired genes affecting the HFs cycle based on the comparative analysis of lncRNA-seq data between the yak and cashmere goat.

\section{Sequence conservation properties analysis of IncRNAs between yak and cashmere goat}

In the above section, the sequence conservation of the 110 DELs in yak was investigated by compared with cashmere goat lncRNA data during HFs cycle using NCBI BLAST-2.9.0+. Then, a detailed analysis of the sequence conservation properties of these aligned lncRNAs were performed. In the result of NCBI BLAST2.9.0+, total of 287 alignments including 332 hits were obtained in goat lncRNA data, which were aligned by 24 DELs of the yak database (Additional file 6) [19]. Length of the matching regions (identities) of the hits was distributed between 36 and $3765 \mathrm{bp}$, and the matching percentage was more than $80 \%$ (Additional file 6). Figure 4a shows the length distribution of the matching sequence of all the hits by BLAST-2.9.0+, which indicates that the number of the matching sequence distributed between 101 and $150 \mathrm{bp}$ is the most, and the matching regions distributed between 51 and 200 accounting for $82 \%$ of all the hits [19] (Fig. 4a, Additional file 5). Interestingly, the analysis of every aligned lncRNA found that most of the queried lncRNAs of the yak had numerous alignments with cashmere goat lncRNA dataset while the matching regions on one lncRNA in the yak were constrained (Additional files 6 and 7). Table 1 presents partial queries that aligned more than 10 lncRNA sequences in cashmere goat dataset. For example, the matching region of lncRNA TCONS_00027937 with a high sequence conservation that aligned to 35 lncRNA sequences of the cashmere goat dataset while all of these alignments roughly matched with the region of 43-160 of TCONS_00027937, the region might be an important element for the function of TCONS_00027937. Then, the lncRNAs TCONS_00008989 and TCONS_00020227 were randomly selected, along with lncRNA TCONS 00016111, which had the longest matching sequence to $3765 \mathrm{bp}$, were used to perform further detailed analysis for their matching regions.

For TCONS_00008989, all the alignments of cashmere goat data were roughly mapped to the region of 243 442 of TCONS_00008989. Then, a local multiple sequence alignment was manually performed in the matching region of TCONS_00008989 with other aligned 11 different sequences in cashmere goat. The result showed that the matching region between 315 and 356 of TCONS_00008989 about $40 \mathrm{bp}$ with high sequence identity among 10 of the alignments (Figure S2). The sequence logo of this highly identical region among alignments was presented using WebLogo3 (http:// weblogo.threeplusone.com/) (Fig. 4b). This short sequence might be an essential binding module for 


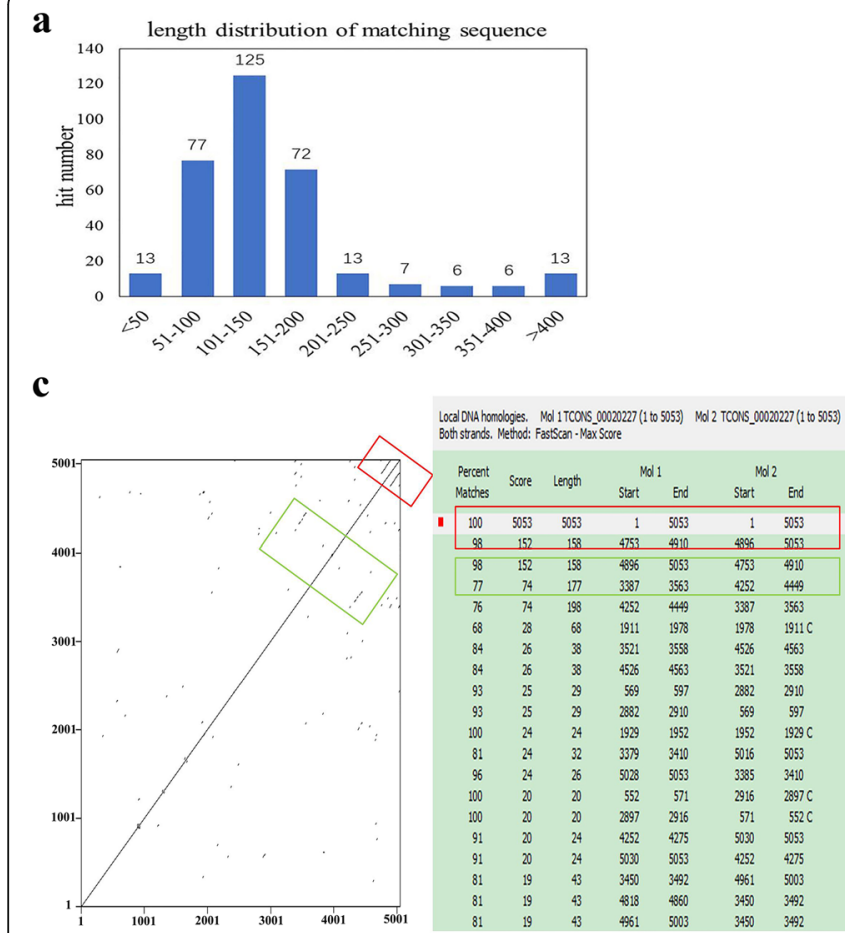

b

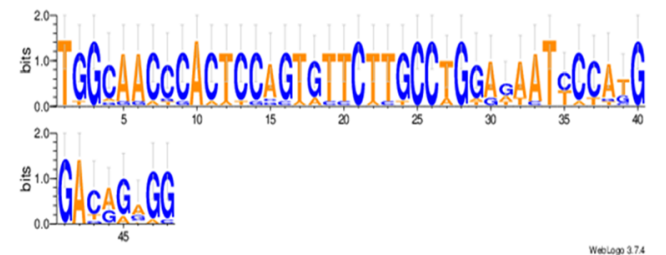

d
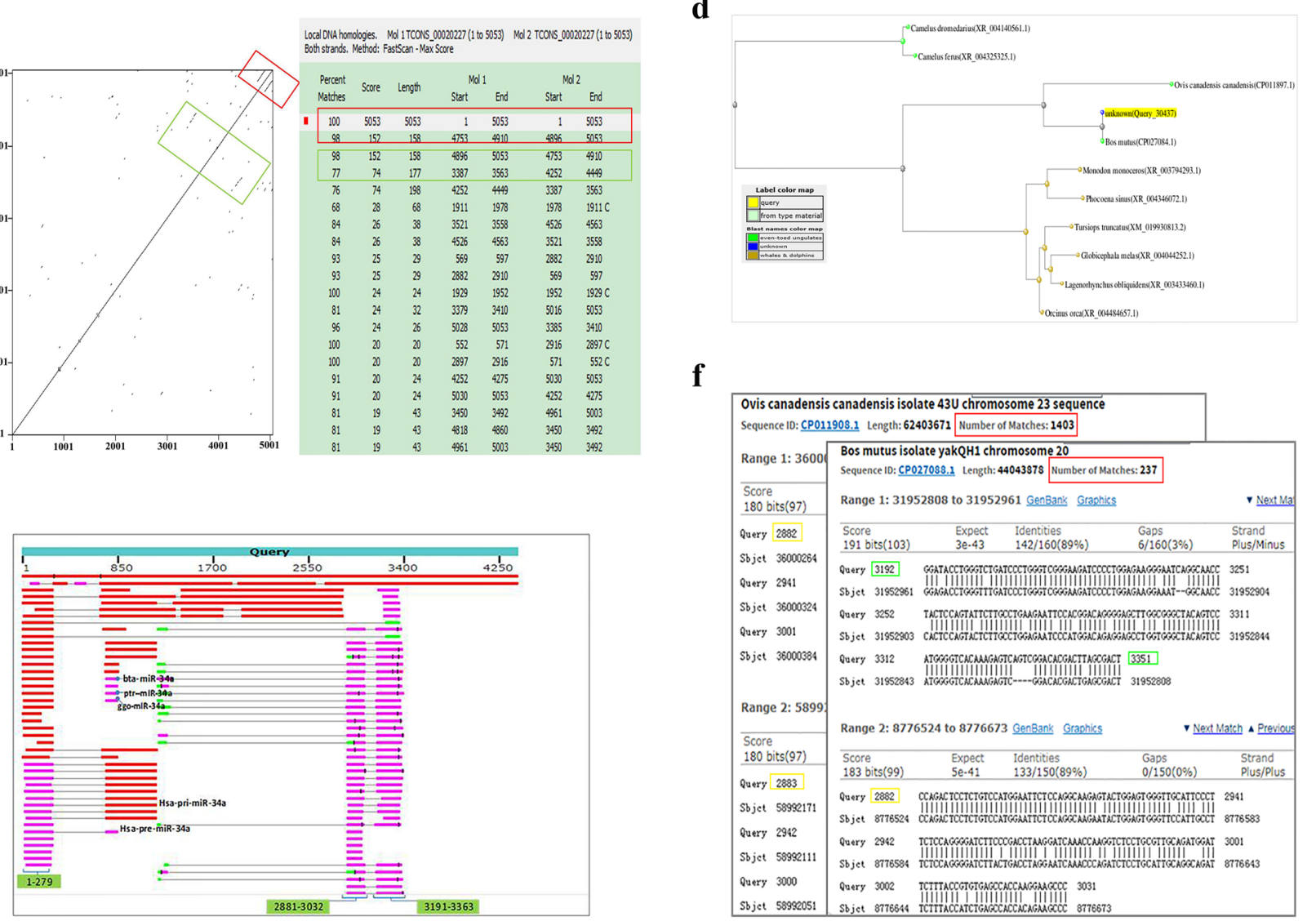

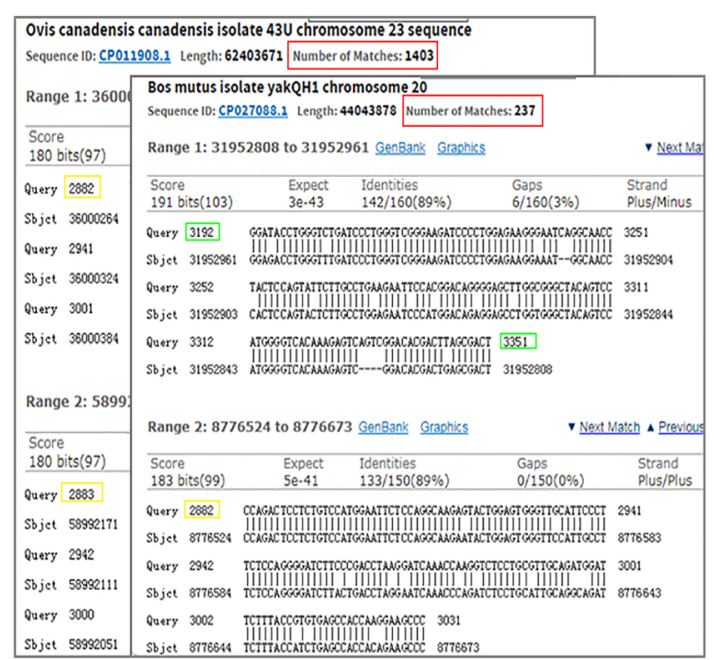

Fig. 4 Analysis of conservation properties of the IncRNA alignments between yak and cashmere goat. a Length distribution of the matching sequence of all the hits by NCBI BLAST-2.9.0+. b Sequence logo of the highly conserved sequences between TCONS_00008989 and its aligned 10 cashmere goat IncRNAs using WebLogo3. c Dot plot of TCONS_00020227 and the detailed ranges of the repeats on TCONS_00020227 were analyzed using dottup website and Clone Manager software, respectively. The boxes with the same color indicate the same region of TCONS_00020227, and the red and green boxes represented two pairs of repeats on TCONS_00020227. d The Blast Tree View of TCONS_00016111 produced using BLAST pairwise alignments with neighbor joining algorithm. Query_55251 presented TCONS_00016111, and other terms on the tree were the first seven sequences of TCONS_00016111 BLAST result. e Graphic summary of selected 100 sequences in defaulted first page of TCONS_00016111 BLAST result. Three matching regions of TCONS_00016111 are marked in green box. f Typical alignments example of the two key matching regions, about 2881-3029 and 3191-3365, in the chromosome of Ovis canadensis canadensis and Bos mutus (yak). The number of matches in each of these two chromosomes is highlighted using red box

dictating the function of TCONS_00008989. Besides, it was speculated that a common feature of sequence conservation of lncRNAs was a short highly conserved sequence shared by multiple lncRNAs of one species or various species.

TCONS_00020227 is an intronic lncRNA, which was aligned to 31 lncRNA sequences of cashmere goat data, mainly matched with 4 regions: 2506-3121, 4251-4452, 4723-4898, and 4886-5053 within TCONS_00020227 (Additional file 6, Table 1). Compared with TCONS 00008989, the matching regions in TCONS_00020227 were relatively varied. The presence of repeats on TCONS_00020227 was analyzed using the Dot Plot of TCONS_00020227 on the dottup website. Two pairs of 
Table 1 Information of DELs aligned with numerous IncRNA sequences of cashmere goat

\begin{tabular}{lllll}
\hline Query & Length & Number of matched alignments & Partner_gene & Regions of matching sequence \\
\hline TCONS_00008989 & 1549 & 13 & GPRC5D & $243-442$ \\
TCONS_00020227 & 5053 & 31 & TMEM231 & $2506-3121,4251-4452,4723-4898,4886-5053$ \\
TCONS_00058749 & 2278 & 25 & ASAH2 & $172-359,1673-1863$ \\
TCONS_00055139 & 2913 & 12 & BACH1 & $920-1463$ \\
TCONS_00033093 & 2364 & 11 & EMX2 & $1929-2091$ \\
TCONS_00018581 & 229 & 14 & FER & $1-229$ \\
TCONS_00016111 & 4400 & 25 & GPR157 & $690-4400(2), 103-279(2), 277-689(1), 2881-3029,3191-3365$ \\
TCONS_00027937 & 6622 & 35 & ISPD & $43-160$ \\
TCONS_00000176 & 839 & 16 & LIPG & $1-133,131-267$ \\
TCONS_00016755 & 1962 & 15 & LOC102274179 & $923-1962$ \\
TCONS_00027432 & 1635 & 30 & OAF & $581-1397(3), 1058-1400,1-171$ \\
TCONS_00031781 & 223 & 15 & COLEC12 & $1-223$ \\
TCONS_00058073 & 3188 & 11 & LOC102267365 & 2867-3059 \\
\hline
\end{tabular}

Note: The number in parentheses refer to the aligned numbers in that region (relative less), and the remaining aligned numbers are assigned to other matching regions

repeats appeared on TCONS_00020227 (Fig. 4c). The detailed regions of the repeats were presented by pairwise alignment using Clone Manager software (Fig. 4c), finding that the latter repeats were coincident with the matching regions of $4723-4898$ and $4886-5053$, detected by NCBI BLAST-2.9.0+ (Fig. 4c and Table 1). The slight difference in the sequence value might be due to the use of distinct analysis methods, but the ranges were comparable. Another repeats appearing in the Dot Plot of TCONS_00020227 were also observed using Clone Manager:4252-4449 (equivalent to 4251-4452 detected by NCBI BLAST-2.9.0+), which repeated with the range of 3387-3563, despite a lower match percentage. This result showed that the matching regions repeatedly existed in TCONS_00020227 and further indicated the importance of the matching sequences. Besides, the data also indicated the effectivity of the BLAST + result.

Finally, the sequence conservation of TCONS 00016111 was analyzed. As TCONS_00016111 had a longer matching region to $3765 \mathrm{bp}$, the sequence conservation of TCONS_00016111 was explored using Nucleotide BLAST in nucleotide collection databases with default parameters. The BLAST result showed that IncRNA TCONS_00016111 mapped to multiple species genomes, including Ovis canadensis canadensis, whales, Bos taurus, Capra hircus. Most of these alignments were ncRNAs (Figure S3). Interestingly, the distance tree of the selected alignments of the top 15 organisms in the defaulted first page of BLAST result showed that TCONS_00016111 with Ovis canadensis canadensis and whales were closer than bovines at the distance tree (Figs. 4d and S3), suggesting a relationship between Ovis canadensis canadensis, whales, and yaks in terms of some evolutionary traits. Moreover, two matching regions 2881-3029 and 3191-3365 of TCONS_0001611 searched using NCBI BLAST-2.9.0+ between yak and cashmere goat lncRNA data emerged in nearly all of the mapped sequences in the first page using Nucleotide BLAST (Fig. 4e). More importantly, these two regions were largely repeated in the genomes of Ovis canadensis Canadensis (bighorn sheep) and Bos mutus (yak) in different chromosomes, the number of repeats was from hundreds to more than one thousand. Figure $4 \mathrm{f}$ shows an example of the searched results. These two regions simultaneously emerged in the genome in abundance, and were found to be partly reverse-complementary so that the region 2881-3365 could form a stable secondary structure (Figure S4), which might be one of the conserved secondary structures of TCONS_0001611. This finding was consistent with the reported conservation properties of lncRNAs in terms of the conserved secondary structure [13]. In addition, miR-34a in cattle, Pan troglodytes, and Gorilla gorilla, and the pre-miR-34a and pri-miR-34a in human were found within the alignments of TCONS_00016111 (Fig. 4e), implicating that TCONS_00016111 also act as a miRNA precursor.

\section{Discussion}

The development of HFs cycle is the characteristic of hair growth in mammals, including anagen, catagen, and telogen [57]. The transition between different phases of this cycle is controlled by the unique regenerating system of follicular epithelial and mesenchymal cells with the interaction of their multiple molecular signals [58]. LncRNAs, as key regulatory molecules have been found to play an important role in the HFs cycle. They have been extensively explored in fur-producing animals due to the economic benefit of animal fibers and observable 
changes in hair growth phenotype $[17,59,60]$. The hair growth pattern of yak is similar to that of cashmere goat, presenting a seasonal phenomenon under the control of photoperiod. This study first investigated the expression profile of lncRNAs during the HFs cycle and analyzed the potential function of DELs on yak. In the HFs cycle, anagen is the longest stage occupying more than half of the whole cycle (1 year) [50]. Division of the HFs cycle in yak has been rarely reported to data. In this study, samples in five time points of yak were employed to perform IncRNA sequencing, including Jan., Mar., Jun., Aug., and Oct.. After a preliminary analysis of the DELs data between the comparison groups, Oct. was selected as the optimum time in the anagen with Jan. (catagen) and Mar. (telogen) for further functional analysis. The samples in the HFs cycle of yak were collected referring to the phase division of a cashmere goat data and based on the phenotypic changes in yak [50]. Previous studies reported that the phase division of the HFs cycle in Shaibei cashmere goat in North China was as follows: the anagen was from Apr. to Oct., the catagen was from Nov. to Jan., and the telogen was from Feb. to Mar.. In this study, DELs between Jan., Mar. and Oct. in the sequencing date were relatively more than those in other comparison groups, and the distinct phases of HFs cycle could be significantly distinguished (Fig. 1c). Jun. might be a transition phase from the telogen to anagen. Oct. had more changes in the anagen phase compared with Jun. and Aug., which was consistent with a previous study reporting that the ratio of secondary to primary follicles $(\mathrm{S} / \mathrm{P})$ in Oct. was the highest in cashmere goat [50]. After confirming the optimum three phases of the HFs cycle in the yak, the functional analysis of the DELs in the three distinct phases were performed.

LncRNAs could affect the gene expression in the immediate genomic vicinity (in cis) $[12,14]$. In the present study, the nearest gene within $100 \mathrm{~kb}$ of lncRNAs was searched and used to predict the potential function of DELs by GO and KEGG enrichment analysis. The result showed that in the transition from the anagen to catagen (Oct.-vs-Jan.), the primarily enriched GO terms of cell communication and metabolism (e.g. cell surface receptor signaling, carbohydrate metabolic process, and protein O-linked mannosylation) were down-regulated, and cell transformation increased (e.g. keratinization and mitotic cell cycle). In the transition from the catagen to the telogen (Jan.-vs-Mar.), the function of nutrition metabolism (e.g. thiamine transport, response to glucose, and regulation of insulin secretion) was mainly enriched, which might be related to the preparation for follicle reset. Interestingly, the functions associated with protein ubiquitination was enriched several times in the transition from the telogen to the anagen
(Mar.-vs-Oct.). Previous studies reported that the ubiquitin-mediated proteolysis pathway was important for distinguishing the SFs from PFs of cashmere goats $[46,47]$, which coincided with the present data that Oct. might be the phase with thriving SFs and the growth of PFs might be vigorous in Mar. [50]. The signaling pathways involved in HFs morphogenesis were important for maintaining the normal periodical cycle of hair growth [5]. KEGG enrichment analysis found that Wnt signaling, which was widely reported playing a curial role in HFs development [48,61], was enriched in the Jan.-vs-Mar. group. The VEGF signaling pathway was enriched in both the Jan.-vs-Mar. and Mar.-vs-Oct. groups. VEGF was involved in the regulation of the HFs cycle by inducing the angiogenesis of dermal papilla [9]. The HIF-1 signaling pathway was enriched in the Mar.-vs-Oct. group, which might be associated with the hypoxia adaptability of yak [62]. From a holistic perspective, the pathways that emerged in the distinct phases seemed to orchestrate a dynamic genesis of the HFs cycle at a molecular level that corresponded with the biological process of HFs development.

A large number of DELs during the HFs cycle in various species were examined following the rapid development of genome sequencing technology, but the identified functional lncRNAs were rare, partly due to the lower level of sequence conservation of IncRNAs across species [12]. The DELs with nearby paired DEGs were screened and the sequence conversation of these lncRNAs between the yak and cashmere goat during HFs cycle was further analyzed to obtain more reliable DELs affecting the HFs growth. Twenty-four DELs were found with sequence conservation between the yak and the cashmere goat lncRNA data to different degrees. Sequence conversation was an important factor in revealing the function of lncRNAs. The wellstudied lncRNAs, such as Xist, HOTAIR, and MALA T1, were reported with higher sequence conversation or conserved domain [25-27], and it was identified that a highly conserved sequence element of lncRNA was essential for its function [63], although a lack of sequence conservation did not directly imply a lack of function [64]. Previous studies reported that lncRNAs were specifically expressed in tissue and species with poor conservation at the sequence level $[11,65]$. In the present study, yak and cashmere goat all belong to the Bovidae family of ruminants. Also, the seasonal cyclic development of HFs in yaks was similar to that in cashmere goats, including anagen, catagen, and telogen, providing a well molding to study the sequence conservation of IncRNAs for the same trait in different species. The NCBI BLAST-2.9.0+ result showed that 24 lncRNAs among 110 DELs of the yak 
were aligned with cashmere goat IncRNA dataset, accounting for $20 \%$ of total queries (Additional file 6). Many well-studied lncRNAs exhibit well-conserved RNA secondary structures [13], or the highly conserved region of a lncRNA contains a functional motif that regulates one biological process [66]. The sequence conservation properties of these aligned lncRNAs were analyzed in detail. A large number of the queried alignments in yak were aligned to multiple lncRNA sequences in cashmere goat dataset, while the matching regions in the queried lncRNAs of yak were constrained; these matching sequences probably contained the elements essential for their activity [67-69]. In the present study, three lncRNAs were selected for further detailed analysis. Among these lncRNAs, TCONS_00008989 aligned to 13 lncRNA sequences of cashmere goat data. All of these alignments nearly matched with one region: about 200 bp of TCONS_00008989, and approximately 40 bp was highly conversed, which might be a key recognition sequence of TCONS_00008989. Another 2 selected lncRNAs TCONS_00020227 and TCONS_00016111 were respectively aligned to 25 and 30 lncRNA sequences in cashmere goat dataset. The matching sequences of TCONS_00020227 were repeatedly observed in TCONS_ 00020227, which might be a commonality of many sequence conserved lncRNAs. TCONS_00016111 was matched with a longer sequence of a cashmere goat lncRNA. Hence, Online BLAST was performed, revealing that the molecular evolution tree of TCONS_00016111 was different from the species tree of yak (http://asia. ensembl.org/info/about/speciestree.html). The distance of yak in this molecular evolution tree was closer to Ovis canadensis canadensis and whales instead of cattle in species tree (Figs. 4d, S3). It was well established that whales were more closely related to cows and their relatives compared with other mammal such as donkeys and horses and their perissodactyl relatives [70]. In addition, the oldest fossil of an approximately 50-million-year-old whale was discovered in the coeval sediments of Indo-Pakistan $[71,72]$. This area was close to the habitat of wild yaks on the Tibetan plateau, which also provided a clue for the hypothesis of an evolutionary relationship between whales and yaks. A transition of lncRNAs was reported from conserved lncRNAs toward an increasing number of lineageand organ- specific lncRNAs during their development [11]. On the other hand, IncRNAs were found to be ancient components in the evolution of vertebrate genome [29], it was believed that a few highly conserved lncRNAs in evolution still existed; for example, the IncRNA TCONS_00016111 found in the BLAST analysis in the present study. Further, notably, two matching regions that could form a stable secondary structure in TCONS 00016111 were largely repeated from hundreds to more than one thousand in the genome of Ovis canadensis canadensis and yak (Figures S4 and 4f), implicating that the repeatedly emerging sequences might act as an important module to recognize RNA, DNA, or protein for the activity on their genomic loci [13, 63]. Meanwhile, these data also indicated the efficiency of the aligned conservation elements using NCBI BLAST-2.9.0 + .

\section{Conclusions}

This study was novel in presenting the data on the lncRNAs of yak during the HFs cycle using lncRNA-seq. The functions of the identified DELs were indirectly predicted via the enrichment analysis of the function of their nearest mRNAs. In addition, several sequence-conserved lncRNAs between yak and cashmere goat in the HFs cycle were searched using NCBI BLAST-2.9.0+, and the sequence conservation properties were analyzed. The conserved sequence elements might play an important role in the regulation of the HFs cycle. The finding of the present study might provide valuable insight into the study of lncRNAs functionality and mechanism in the HFs cycle.

\section{Supplementary information}

Supplementary information accompanies this paper at https://doi.org/10. 1186/s12864-020-07082-z.

\section{Additional file 1. \\ Additional file 2. \\ Additional file 3. \\ Additional file 4. \\ Additional file 5. \\ Additional file 6. \\ Additional file 7 \\ Additional file 8. \\ Additional file 9. \\ Additional file 10 . \\ Additional file 11. \\ Additional file 12 .}

\section{Abbreviations}

DEGs: Differentially expressed genes; DELs: Differentially expressed IncRNAs; HFs: Hair follicles; KEGG: Kyoto Encyclopedia of Genes and Genomes; IncRNAs: Long non-coding RNAs

\section{Acknowledgements}

We thank the Shanghai OE biotech Co., Ltd. (Shanghai, China) for the transcriptome sequencing and analysis.

\section{Declarations}

All authors reviewed and approved the final manuscript.

\section{Authors' contributions}

XZ analyzed the data and wrote the article. QB and CJ contributed significantly to bioinformatics analysis and visualized the data. CL1 and YC extracted the RNA and performed the RT-qPCR. XW and CL2 collected the skin samples. PB and PY conceived the study, designed the experiment, interpreted the results, and revised the manuscript. All authors read and approved the manuscript. 


\section{Funding}

This study was supported with four funders. The Agricultural Science and Technology Innovation Program [CAAS-ASTIP-2014-LIHPS-01] provided the sampling funding, the National Beef Cattle Industry Technology \& System [CARS-37] provided the source of experimental animals, National Natural Science Foundation of China [31660636] and the Central Public-interest Scientific Institution Basal Research Fund [1610322017003] provided the sequencing funding and laboratory verification cost. The funding bodies played no role in the design of the study and collection, analysis, and interpretation of data and in writing the manuscript.

\section{Availability of data and materials}

The datasets generated and analysed during the current study are available in the NCBI repository, accession number: PRJNA550233 (https://www.ncbi. nlm.nih.gov/bioproject/PRJNA550233).

The data supporting the conclusions of this study are available within the additional files.

\section{Ethics approval and consent to participate}

All procedures involving animals were performed according to the guidelines of the China Council on Animal Care and the Ministry of Agriculture of the People's Republic of China and approved by the Animal Ethics Committee of Lanzhou Institute of Husbandry and Pharmaceutical Sciences, Chinese Academy of Agricultural Sciences.

\section{Consent for publication}

Not applicable.

\section{Competing interests}

The authors declare no competing interests.

\section{Received: 24 February 2020 Accepted: 18 September 2020}

Published online: 01 October 2020

\section{References}

1. Kloren W, Norton BW, Waters MJ. Fleece growth in Australian cashmere goats. III. The seasonal patterns of cashmere and hair growth, and association with growth hormone, prolactin and thyroxine in blood. Crop Pasture Sci. 1993;44(5):1035-50.

2. Mcdonald B, Hoey W, Hopkins P. Cyclical fleece growth in cashmere goats. Aust J Agric Res. 1987;38(3):597.

3. Baker RE, Murray PJ. Understanding hair follicle cycling: a systems approach. Curr Opin Genet Dev. 2012;22(6):607-12.

4. Alonso L, Fuchs E. The hair cycle. J Cell Sci. 2006;119(Pt 3):391-3.

5. Rishikaysh P, Dev K, Diaz D, Qureshi W, Filip S, Mokry J. Signaling involved in hair follicle morphogenesis and development. Int J Mol Sci. 2014;15(1): 1647-70.

6. Oh HS, Smart RC. An estrogen receptor pathway regulates the telogenanagen hair follicle transition and influences epidermal cell proliferation. P Natl Acad Sci. 1996;93(22):12525-30

7. Ibraheem M, Galbraith H, Scaife J, Ewen S. Growth of secondary hair follicles of the cashmere goat in vitro and their response to prolactin and melatonin. J Anat. 1994;185(Pt 1):135-42.

8. Foitzik K, Lindner G, Mueller-Roever S, Maurer M, Botchkareva N, Botchkarev $\checkmark$, Handjiski B. Control of murine hair follicle regression (catagen) by TGFbeta1 in vivo. FASEB J. 2000;14(5):752-60.

9. Castexrizzi N, Lachgar S, Charveron M, Gall Y. Implication of VEGF, steroid hormones and neuropeptides in hair follicle cell responses. Ann Dermatol Vener. 2002;129(5 Pt 2):783-6.

10. Guttman M, Rinn JL. Modular regulatory principles of large non- coding RNAs. Nature. 2012;482(7385):339-46.

11. Sarropoulos I, Marin R, Cardoso-Moreira M, Kaessmann H. Developmental dynamics of IncRNAs across mammalian organs and species. Nature. 2019; 571(7766):510-4

12. Amaral PP, Leonardi T, Han N, Viré E, Gascoigne DK, Arias-Carrasco R, Büscher M, Pandolfini L, Zhang A, Pluchino S. Genomic positional conservation identifies topological anchor point RNAs linked to developmental loci. Genome Biol. 2018;19(1):32.

13. Nitsche A, Stadler PF. Evolutionary clues in IncRNAs. Wiley Interdiscip Rev RNA. 2017;8(1):e1376.
14. Roberts TC, Morris KV, Weinberg MS. Perspectives on the mechanism of transcriptional regulation by long non-coding RNAs. Epigenetics. 2014;9(1): 13-20.

15. Mitchell G, Julie D, Bryce WC, Manuel G, Jennifer KG, Glen M, Geneva Y, Anne Bergstrom L, Robert A, Laurakay B, et al. lincRNAs act in the circuitry controlling pluripotency and differentiation. Nature. 2011:477(7364):295-300.

16. Lin CM, Liu Y, Huang K. Chen X-c, Cai B-z, Li H-h, Yuan Y-p, Zhang H, Li Y. long noncoding RNA expression in dermal papilla cells contributes to hairy gene regulation. Biochem Bioph Res Co. 2014;453(3):508-14.

17. Zhao B, Chen Y, Hu S, Yang N, Wang M, Liu M, Li J, Xiao Y, Wu X. Systematic Analysis of Non-coding RNAs Involved in the Angora Rabbit (Oryctolagus cuniculus) Hair Follicle Cycle by RNA Sequencing. Front Gene. 2019;10(407): eCollection 2019.

18. Sulayman A, Tian K, Huang X, Tian Y, Xu X, Fu X, Zhao B, Wu W, Wang D, Tulafu AYH. Genome-wide identification and characterization of long noncoding RNAs expressed during sheep fetal and postnatal hair follicle development. Sci Rep. 2019:9(1):8501.

19. Wang S, Wei G, Zhixin L, Yang G, Beilei J, Lei Q, Zhiying Z, Xin W. Integrated analysis of coding genes and non-coding RNAs during hair follicle cycle of cashmere goat (Capra hircus). BMC Genomics. 2017;18(1):767.

20. Weikard R, Hadlich F, Kuehn C. Identification of novel transcripts and noncoding RNAs in bovine skin by deep next generation sequencing. BMC Genomics. 2013;14:798.

21. Song LL, Cui Y, Yu SJ, Liu PG, Liu J, Yang X, He JF, Zhang Q. Expression characteristics of BMP2, BMPR-IA and noggin in different stages of hair follicle in yak skin. Gen Comp Endocr. 2018;260:18-24.

22. Song LL, Cui Y, Xiao L, Yu SJ, He JF. DHT and E2 synthesis-related proteins and receptors expression in male yak skin during different hair follicle stages. Gen Comp Endocr. 2020;286:113245.

23. Song LL, Cui Y, Yu SJ, Liu P-G, He JF. TGF-beta and HSP70 profiles during transformation of yak hair follicles from the anagen to catagen stage. J Cell Physiol. 2019;234(9):15638-46.

24. Jia C, Wang H, Li C, Wu X, Zan L, Ding X, Guo X, Bao P, Pei J, Chu M, et al. Genome-wide detection of copy number variations in polled yak using the Illumina BovineHD BeadChip. BMC Genomics. 2019;20(1):376.

25. Gupta RA, Shah N, Wang KC, Kim J, Horlings HM, Wong DJ, Tsai M-C, Hung T, Argani P, Rinn JL, et al. Long non-coding RNA HOTAIR reprograms chromatin state to promote cancer metastasis. Nature. 2010:464(7291):10716.

26. Gutschner T, Hämmerle M, Diederichs S. MALAT1 —a paradigm for long noncoding RNA function in cancer. J Mol Med. 2013;91(7):791-801.

27. Brown CJ, Hendrich $B D$, Rupert JL, Lafrenière RG, Xing Y, Lawrence J, Willard HF. The human XIST gene: analysis of a $17 \mathrm{~kb}$ inactive X-specific RNA that contains conserved repeats and is highly localized within the nucleus. Cell. 1992;71(3):527-42.

28. Jeon $Y$, Lee JT. $Y Y 1$ tethers Xist RNA to the inactive $X$ nucleation center. Cell. 2011;146(1):119-33.

29. Nitsche A, Rose D, Fasold M, Reiche K, Stadler PF. Comparison of splice sites reveals that long noncoding RNAs are evolutionarily well conserved. RNA. 2015;21(5):801-12

30. Johnsson P, Lipovich L, Grandér D, Morris KV. Evolutionary conservation of long non-coding RNAs; sequence, structure, function. Biochim Biophys Acta. 2014;1840(3):1063-71.

31. Hezroni H, Koppstein D, Schwartz MG, Avrutin A, Bartel DP, Ulitsky I. Principles of long noncoding RNA evolution derived from direct comparison of Transcriptomes in 17 species. Cell Rep. 2015;11(7):1110-22.

32. Kim D, Langmead B, Salzberg SL. HISAT: a fast spliced aligner with low memory requirements. Nat Methods. 2015;12(4):357-60.

33. Trapnell C, Roberts A, Goff L, Pertea G, Kim D, Kelley DR, Pimentel H, Salzberg SL, Rinn JL, Pachter L. Differential gene and transcript expression analysis of RNA-seq experiments with TopHat and cufflinks. Nat Protoc. 2012;7(3):562-78.

34. Pertea M, Pertea GM, Antonescu CM, Chang TC, Mendell JT, Salzberg SL. StringTie enables improved reconstruction of a transcriptome from RNA-seq reads. Nat Biotechnol. 2015;33(3):290-5.

35. Liang S, Luo H, Bu D, Zhao G, Yu K, Zhang C, Liu Y, Chen R, Yi Z. Utilizing sequence intrinsic composition to classify protein-coding and long noncoding transcripts. Nucleic Acids Res. 2013:41(17):e166.

36. Lei $K$, Yong Z, Ye ZQ, Liu XQ, Zhao SQ, Wei L, Ge G. CPC: assess the proteincoding potential of transcripts using sequence features and support vector machine. Nucleic Acids Res. 2007;35(Web Server issue):W345-9. 
37. Finn RD, Bateman A, Clements J, Coggill P, Eberhardt RY, Eddy SR, Heger A, Hetherington K, Holm L, Mistry J. Pfam: the protein families database. Nucleic Acids Res. 2014;42(D1):D222-30.

38. Li A, Zhang J, Zhou Z. PLEK: a tool for predicting long non-coding RNAs and messenger RNAs based on an improved k-mer scheme. BMC Bioinformatics. 2014;15(1):311.

39. Valentin W, Fabrice L, Benoît H, Guillaume R, Lætitia L, Tosso L, Vidhya J, Edouard C, Audrey D, Hannes L. FEELnc: a tool for long non-coding RNA annotation and its application to the dog transcriptome. Nucleic Acids Res. 2017:45(8):e57.

40. Trapnell C, Williams BA, Pertea G, Mortazavi A, Kwan G, van Baren MJ, Salzberg SL, Wold BJ, Pachter L. Transcript assembly and quantification by RNA-Seq reveals unannotated transcripts and isoform switching during cell differentiation. Nat Biotechnol. 2010;28(5):511-5.

41. Love Ml, Huber W, Anders S. Moderated estimation of fold change and dispersion for RNA-seq data with DESeq2. Genome Biol. 2014;15(12):550.

42. $Y u$ G, Wang LG, Han Y, He QY. clusterProfiler: an R package for comparing biological themes among gene clusters. Omics. 2012;16(5):284-7.

43. Bao P, Luo J, Liu Y, Chu M, Ren Q, Guo X, Tang B, Ding X, Qiu Q, Pan H, et al. The seasonal development dynamics of the yak hair cycle transcriptome. BMC Genomics. 2020;21(1):355

44. Camacho CG, Coulouris G, Avagyan V, Ma N, Papadopoulos J, Bealer K, Madden TL. BLAST+: architecture and applications. BMC Bioinformatics. 2009;10:421.

45. Zhang S, Qin C, Cao G, Xin W, Feng C, Zhang W. Systematic analysis of long noncoding RNAs in the senescence-accelerated mouse prone 8 brain using RNA sequencing. Mol Ther Nucleic Acids. 2016;5(8):e343.

46. Ji XY, Wang JX, Liu B, Zheng ZQ, Fu SY, Mekuriaw TG, Bai X, Bai YS, Li H, Zhang WG. Comparative Transcriptome analysis reveals that a ubiquitinmediated proteolysis pathway is important for primary and secondary hair follicle development in cashmere goats. PLoS One. 2016;11(10):e0156124.

47. Huntzicker EG, Oro AE. Controlling hair follicle signaling pathways through Polyubiquitination. J Invest Dermatol. 2008;128(5):1081-7.

48. Tsai SY, Sennett R, Rezza A, Clavel C, Grisanti L, Zemla R, Najam S, Rendl M $W n t / \beta$-catenin signaling in dermal condensates is required for hair follicle formation. Dev Biol. 2014;385(2):179-88.

49. Yamamoto N, Tanigaki K, Han H, Hiai H, Honjo T. Notch/RBP-J signaling regulates epidermis/hair fate determination of hair follicular stem cells. Curr Biol. 2003;13(4):333-8.

50. Chao L, Li Y, Zhou GX, Gao Y, Ma S, Chen YL, Song JZ, Wang XL. Wholegenome bisulfite sequencing of goat skins identifies signatures associated with hair cycling. BMC Genomics. 2018;19(1):638.

51. Nyberg KG, Machado CA, Notes A. Comparative expression dynamics of Intergenic long noncoding RNAs in the genus drosophila. Genome Biol Evol. 2016;8(6):1839-58.

52. Infante P, Severini LL, Bernardi F, Bufalieri F, Marcotullio LD. Targeting hedgehog Signalling through the Ubiquitylation process: the multiple roles of the HECT-E3 ligase Itch. Cells. 2019;8(2):98

53. Suen WJ, Li ST, Yang LT. Hes1 regulates anagen initiation and hair follicle regeneration through modulation of hedgehog signaling. Stem Cells. 2020; 38(2):301-14.

54. Massa F, Tammaro R, Prado MA, Cesana M, Lee BH. The deubiquitinating enzyme USP14 controls ciliogenesis and hedgehog signalling. Hum Mol Genet. 2018;28(5):764-77.

55. Gao $Y$, Wang $X$, Yan H, Zeng J, Ma S, Niu Y, Zhou G, Jiang Y, Chen $Y$. Comparative Transcriptome analysis of fetal skin reveals key genes related to hair follicle morphogenesis in cashmere goats. PLoS One. 2016;11(3): e0151118.

56. Jacobo A, Dasgupta A, Erzberger A, Siletti K, Hudspeth AJ. Notch-mediated determination of hair-bundle polarity in Mechanosensory hair cells of the Zebrafish lateral line. Curr Biol. 2019;29(21):3579-87.

57. Stenn KS, R. P: controls of hair follicle cycling. Physiol Rev. 2001;81(1):449-94

58. Sennett R, Rendl M. Mesenchymal-epithelial interactions during hair follicle morphogenesis and cycling. Semin Cell Dev Biol. 2012;23(8):917-27.

59. Jiao $Q$, Yin RH, Zhao SJ, Wang ZY, Zhu YB, Wang W, Zheng YY, Yin XB, Guo D, Wang SQ, et al. Identification and molecular analysis of a IncRNA-HOTAIR transcript from secondary hair follicle of cashmere goat reveal integrated regulatory network with the expression regulated potentially by its promoter methylation. Gene. 2019;688:182-92.

60. Wei G, Wang SH, Sun B, Zhang YL, Shen W, Hasan K. Melatonin promotes cashmere goat (Capra hircus) secondary hair follicle growth: a view from integrated analysis of long non-coding and coding RNAs. Cell Cycle. 2018; 17(10):1255-67.

61. Mater DV, Kolligs FT, Dlugosz AA, Fearon ER. Transient activation of Beta -catenin signaling in cutaneous keratinocytes is sufficient to trigger the active growth phase of the hair cycle in mice. Genes Dev. 2003;17(10):121924.

62. Ivan M, Kondo K, Yang H, Kim W, Valiando J, Ohh M, Salic A, Asara JM, Lane WS, Kaelin WG. HIFalpha targeted for VHL-mediated destruction by Proline hydroxylation: implications for O2 sensing. Science. 2001;292(5516):464-8.

63. Marín-Béjar O, Mas AM, González J, Martinez D, Athie A, Morales X, Galduroz M, Raimondi I, Grossi E, Guo S. The human IncRNA LINC-PINT inhibits tumor cell invasion through a highly conserved sequence element. Genome Biol. 2017;18(1):202.

64. Poliseno L, Salmena L, Zhang J, Carver B, Haveman WJ, Pandolfi PP. A coding-independent function of gene and pseudogene mRNAs regulates tumour biology. Nature. 2010;465(7301):1033-8.

65. Zheng H, Li J, Liu D, Li H, Samudrala R, Yu J, Wong GK-S, Wang J, Zhang J. Mouse transcriptome: neutral evolution of 'non-coding' complementary DNAs. Nature. 2004;431(7010):1-757.

66. Lin N, Chang KY, Li Z, Gates K, Rana TM. An evolutionarily conserved long noncoding RNA TUNA controls Pluripotency and neural lineage commitment. Mol Cell. 2014:53(6):1005-19.

67. Ulitsky I. Evolution to the rescue: using comparative genomics to understand long non-coding RNAs. Nat Rev Genet. 2016;17(10):601.

68. Chen J, Shishkin AA, Zhu X, Kadri S, Maza I, Guttman M, Hanna JH, Regev A, Garber M. Evolutionary analysis across mammals reveals distinct classes of long non-coding RNAs. Genome Biol. 2016;17(1):19.

69. Necsulea A, Soumillon M, Warnefors M, Liechti A, Daish T, Zeller U, Baker JC, Gruetzner F, Kaessmann $H$. The evolution of IncRNA repertoires and expression patterns in tetrapods. Nature. 2014;505(7485):635-40 a611.

70. Meyer A. From donkeys and cows to whales. Nature. 2000;406(6797):677-8.

71. Thewissen JGM, Cooper LN, Clementz MT, Bajpai S, Tiwari BN. Whales originated from aquatic artiodactyls in the Eocene epoch of India. Nature. 2007:450(7173):1190-4.

72. Geisler JH. Whale evolution: dispersal by paddle or fluke. Curr Biol. 2019; 29(8):R294-6.

\section{Publisher's Note}

Springer Nature remains neutral with regard to jurisdictional claims in published maps and institutional affiliations.

\section{Ready to submit your research? Choose BMC and benefit from:}

- fast, convenient online submission

- thorough peer review by experienced researchers in your field

- rapid publication on acceptance

- support for research data, including large and complex data types

- gold Open Access which fosters wider collaboration and increased citations

- maximum visibility for your research: over $100 \mathrm{M}$ website views per year

At BMC, research is always in progress.

Learn more biomedcentral.com/submissions 